\title{
Doppia oscillazione diurna della pressione atmosferica e del gradiente verticale del campo elettromagnetico
}

(Double diurnal oscillation of the atmospheric pressure and of the vertical gradient of the electro-atmospheric field)

\author{
R. JANESELLI $(*)$
}

Ricevuto il 24 Giugno 1968

\begin{abstract}
Rrassusto. -- Riferite le caratteristiche essenziali circa la doppia oseillazione diurna della pressione atmosferica sopra tutta la superficie terrestre, usufruendo dei barogrammi forniti dall'Osservatorio Metereolorico dell'Istituto Cavanis di Venezia, vengono tracciati i diagrammi delle medie orarie per i singoli mesi del novennio 1959-1967. I medesimi diagrammi mensili ed annuali vengono costruiti per il fradiente verticale del campo elettro-atmosferico del quinquennio 1963-1967. Il parallelismo tra le due serie di diagrammi risulta buono, quasi perfetto tra i due diagrammi, cho comprendono tutte le misure prese in considerazione. Quindi si fa un breve cenno intorno alla struttura del campo elettrico dell'atmosfera terrestre, gnale ci viene fornita dalle ultime scoperte conseguite per mezo dei razzi meteorologici e satelliti artificiali. si conclucle facendo osservare che molto probabilmente la doppia oscillazione delle due grandezze considerate c̀ pro. dotta dalle medesime canse, specialmente dalle maree atmosferiche e dai campi elettrici da esse generati.
\end{abstract}

Sumary. - After having stated the essential characteristies about the double diurnal oscillation of the atmospheric pressure over the whole Earths surface, using the barograms of the metereologieal Ohservatory of Istituto Cavanis in Venice, there are drawn the diagrams of the hour-arerages for the single months from the year 1959 to the year 1967.

The same monthly and ammual diagrams are drawn for the vertical gradient of the electro-atmospheric field for the period 1963-1967.

The parallelism between the two series of diagrams is good, and alnost perfect between the two diagrams which contain all the considered measures. Then there is a short mention about the structure of the electricatmospherie field of the Earth, as it is furnished by the last discoveries, reached by the meteorological rockets and by the artificial satellites. As

$\left({ }^{*}\right)$ Osservatorio Meteorologico dell'Istituto Cavanis di Venezia. 


\section{ERRATA CORRIGE}

Nella nota pubblicata nel Fasc. 3-1968: Vol. XXI pag. 305 di R. Janeselli nel titolo va fatta la seguente correzione:

"Doppia oscillazione diurna della pressione atmosferica e del gradiente verticale del campo elettro-atmosferico". 
a conclusion there is noticed that, with much of probahility, the double oscillation of the two parameters considered is produced by the same causes, especially by the atmospheric tides and by the electric fields, produced by the same tides.

I naturale pensare che come esistono le maree d'acqua (le maree oceaniche), cosi esistano le maree d'aria (le maree atmosferiche). E l'intuizione ci porterebbe a pensare che l'andamento di quest'ultime sia simile a quello delle prime. Però le misure della pressione atmosferica ci hamno rivelato che esistono si le maree atmosferiche, ma che esse hanno un andamento giornaliero del tutto diverso da quelle oceaniche.

Il primo a mettere in evidenza le maree atmosferiche è stato il Laplace, che misurò la pressione atmosferica a Parigi quattro volte al giorno per otto anni. Egli ed i suoi successori trovarono una variazione diurna abbastanza regolare. Migliori misure di quelle di Taplace furono ottenute ai tropici, dove la pressione atmosferica mantiene un andamento molto regolare per lunghi periodi.

Dal tempo di Taplace in poi fu raccolto un materiale immenso di misure sulle variazioni barometriche al livello del mare da stazioni distribuite su tutta la superficie terrestre. Tali registrazioni rivelano, con approssimazione, due massimi alle ore 10 e 22 e due minimi alle ore $t$ e $16\left(^{*}\right)$.

In altre parole le maree atmosferiche seguono il ciclo solare di 12 ore e non quello lunare di 12 ore e 25,5 minuti. L'oscillazione di 12 ore ha attirato il più grande interesse negli ultimi cento anni.

Generalmente l'ampiezza dell'oscillazione durante il giorno è più accentuata di quella che si presenta durante la notte, cosicché la differenza tra il massimo delle ore 10 ed il minimo delle 16 può essere considerata come l'ampiezza della oscillazione durante le 24 ore ( ${ }^{1}$ ). Ordinariamente, nelle regioni a bassa latitudine, naturalmente con tempo bello i massimi ed i minimi, si presentano alle ore suindicate, però l'ampiezza dell'oscillazione diurna varia notevolmente nei diversi mesi dell'anmo, giacché negli equinozi presenta valori più elevati che nei solstizi. Anche a Venezia la massima ampiezza di circa 2 torr, si manifesta agli equinozi. Da ciò deriva che l'ampiezza di tale oscillazione dipende dalla posizione della Terra sulla sua orbita rispetto al sole. locale.

(*) In questo articolo lo ore sono sempre misurate secondo il tempo 
Con l'aumento della latitudine l'ampiezza dell'oscillazione diminuisce gradatamente per ridursi quasi a zero nelle regioni polari. Le diverse condizioni geografiche, topografiche e climatologiche influiscono moltissimo sulla forma della doppia oscillazione diuma, spostando in modo notevole il massimo delle $10^{\text {n }}$ ed il minimo delle $16^{\mathrm{h}}$. Per es. nelle pianure presentanti un clima continentale, almeno in certi casi, il massimo delle $10^{\mathrm{h}}$ viene anticipato alle $8^{\mathrm{n}}$, mentre il minimo delle $16^{\mathrm{h}}$ viene posticipato alle $17^{\mathrm{h}}$; sulle coste marittime il massimo delle $10^{\mathrm{h}}$ viene posticipato alla $12^{\mathrm{h}}$ ed il minimo delle $16^{\mathrm{h}}$ alle $18^{\mathrm{h}}$ circa. Sul monte Rosa il massimo delle $1^{\mathrm{h}}$ ed il minimo delle $16^{\mathrm{h}}$ si spostano

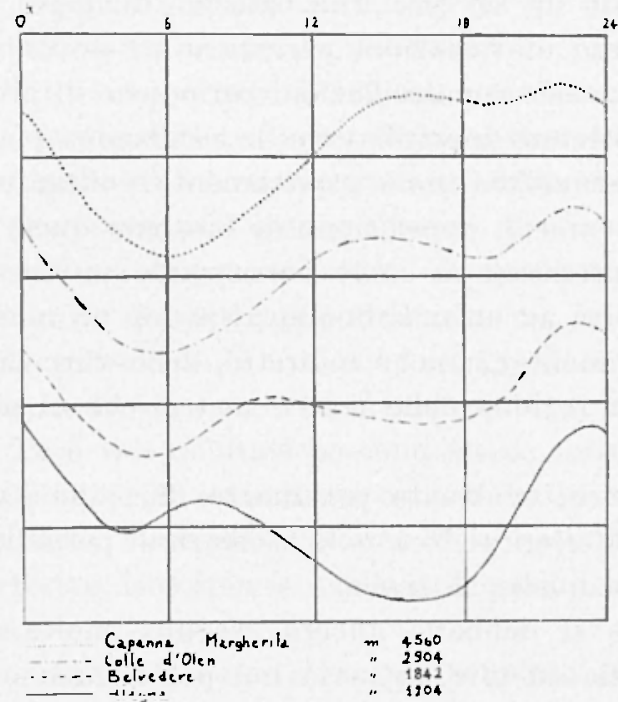

Fig. 1 - Influenza dell'altezza sulla curva giornaliera della pressione atmosferica al Monte Rosa.

in modo evidente in dipendenza dell'altezza: alla capanna Margherita (m. 4560) il massimo del mattino si presenta verso le $16^{\mathrm{h}}$, mentre il minimo del pomeriggio viene molto attenuato (quasi amullato) e ha luogo alle $19^{\mathrm{h}}$ circa; ad Alagna (m. 1204) il massimo delle $10^{\mathrm{h}}$ si presenta intorno alle $7 \frac{12^{h}}{}$ ad il minimo delle $16^{\mathrm{h}}$ verso le $15^{\mathrm{h}}$ (Fig. 1). Invece, in tutte le stazioni suindicate, il minimo del mattino ripresenta sempre tra le ore 4 e le 6 , il massimo della sera tra le 22 e le 24 .

Le maree atmosferiche possono essere rivelate oltre che da misure barometriche, anche da registrazioni effettuate con altri metodi. Uno dei più recenti è lo studio delle tracce meteoriche: cioè lo studio della 
scia, che lascia dietro di sè un meteorita che piomba nell'atmosfera terrestre $\left({ }^{2}\right)$. Per mezzo di tecniche - radar, che sfruttano gli echi delle tracce meteoriche, è possibile calcolare la velocità del vento in prossimita delle scie meteoriche. Il metodo ci fornisce informarioni molto interessanti sulla velocità dei venti ad altezze comprese tra $i$ 75 e $100 \mathrm{~km}$. In particolare tale metodo fu impiegato con successo da Leonard Huxlej e dai suoi collaboratori all'Università di Adelaide in Australia e da Stanlej Greenhow nei laboratori radio-astronomici di Suflicld a Jodrell Bank in Inghilterra. Le misure radar, tra le altre cose, mostrano che a tali altezze esistono dei sistemi di venti periodici aventi un periodo di 12 ore. Tali sistemi di venti possono essere spiegati per mezzo di variazioni periodiche di pressione e quindi essere messi in relazione con oscillazioni periodiche di pressione al suolo.

I risultati ottenuti mostrano che le oscillazioni periodiche di pressione nell'alta atmosfera sono strettamente collegate con quelle al suolo, ma si trovano in opposizione di fase con quest'ultime; cioè ad un minimo di pressione al suolo corrisponde un massimo a grande altezza, e viceversa ad un massimo corrisponde un minimo.

Misure sperimentali, anche indirette, delle variazioni di pressione tra il suolo e la regione delle tracce meteoriche si possono ottenere difficilmente.

Alcume misure, effettuate per mezzo di palloni sonda, mostrano che ad altezze inferiori ai $25 \mathrm{~km}$ le oscillazioni periodiche di pressione sono in fase con quelle al suolo.

Quantunque si debbano ancora eseguire molte osservazioni ad altezze intermedie ed alte, tuttavia noi possediamo attualmente una grande quantità di osservazioni di grande valore, che devono essere spiegate per mezzo di una teoria delle maree atmosferiche.

Tai tempi di Laplace fino ad oggri furono proposte molte ipotesi per spiegare la doppia oscillazione diurna della pressione atmosferica. Pero, nonostante gli sforzi di molti illustri scienziati, dobbiamo dire rha il problema non sembra ancora completamente risolto. Per cui possiamo affermare che la soluzione di tale fenomeno periodico costituisca uno dei problemi più complessi proposti ai geofisici. Non credo opportuno fare la storia di tutte le ipotesi, che sono state proposte per la soluzione di tale problema, perchè di ciò hamno trattato altri scienziati, tra cui Antonino Palumbo in una recente Memoria dal titolo "Sulle Maree Atmosferiche " $\left(^{3}\right)$.

Areennero solo alla famosa teoria della risonanza di Lord Kelvin, perché essa è quella che ha incontrato fino ad oggi il maggior favore. 
Leggendo quanto hamno scritto alcuni scienziati intorno alla teoria della risonanza di Kelvin, mi pare che qualcuno di essi abbia un po" travisato il suo pensiero, per cui ho creduto necessario leggere molto attentamente il suo articolo "On the Acceleration of the Farth's Rotation" ('). Mi sembra di poter sintetizare il suo pensiero nella maniera seguente: "La causa principale della doppia oscilazione diurna della pressione atmosferica non può essere l'attrazione solare o lunare, perché in tal caso il periodo di detta oscillazione dolumare vrebbe essere di 12 ore e 25,5 minuti, essendo l'attrazione (di marea) prevalente su quella solare. Quindi pare certo che la causa principale di tale doppia oscillazione diurna sia da attribuire al calore solare. Ma ovviamente il calore solare dovrebbe produrre una variazione periodica di 24 ore e non di 12, come avviene in realta. Quindi è necessario ammettere che alla oscillazione periodica di $2+$ ore si sovrapponga un'altra di 12 ore, provocata dall'attrazione solare. Per un fenomeno di risonanza l'oscillazione di 12 ore dovrebbe venire amplificata in maniera enorme a scapito di quella di 24 ore.

Inoltre Margules dimostrò più tardi che tra le oscillazioni libere, dell'atmosfera terrestre ve n'ò una con un periodo eguale, con grande approssimazione, a 12 ore; mentre invece nessuma ha un periodo vicino a 24 ore. Però tali risultati possono essere sottoposti a serie critiche, in quanto che egli introdusse nei suoi calcoli delle condizioni molto semplificatrici: trascurò l'effetto dei moti verticali, suppose l'atmosfera terrestre isoterma e considerò pure isoterme le oscillazioni di marea.

Fin dai primi anni del nostro secolo la teoria della risonanza incontrò severe critiche da parte di varii scienziati, tra cui ricordo il Prof. Luigi De Marchi $\left(^{5}\right)$. Secondo quest'ultimo il punto più debole della teoria consiste nel fatto di dare un significato fisico reale ai termini dello sviluppo besseliano della temperatura: infatti tale sviluppo non ì che un semplice artificio analitico. Anche una funzione non periodica, sotto certe condizioni, può essere espressa mediante una serie di Fourier; ma non è lecito "considerare la variazione da essa espressa come somma di tante variazioni armoniche realmente esistenti".

Anche in questi ultimi anni i due scienziati Australiani Buttler e Small hanno dimostrato che la teoria di Lord Kelvin è assolutamente inaccettabile. Secondo Buttler e Small (6), per mezzo delle conoscenze, che attualmente possediamo intorno alla temperatura e alla densità dell'atmosfera terrestre, noi siamo in grado di calcolare, in maniera accurata, gli effetti di marea delle forze gravitazionali solari e lunari. 
Secondo i calcoli di detti fisici, esiste si qualche amplificazione della marea gravitazionale semidiurna solare, ma tale amplificazione dovrebbe essere moltiplicata solo per 2 inveco che per 100 , come sarebbe richiesto per metter d'acrordo le misure sperimentali con i calcoli teorici. E quindi difficile sfuggire alla conclusione che gli efletti gravitazionali sulle maree sono estremamente piccoli, quasi trascurabili ( $\left.{ }^{2}\right)$.

Secondo Buttler e Small $\left(^{6}\right)$, le osciliazioni diurne della pressione atmosferica aventi il periodo di 12 ore, si potrebbero spiegare per mezzo dell'assorbimento della radiazione ultravioletta da parte dell'ozono. Secondo tale ipotesi la ozonosfera esposta ai raggi solari, assorbendo la radiazione ultravioletta, si espande specialmente verso l'alto; il che, ad una certa altezza, dà origine alla formazione di un gradiente di pressione tra la ozonosfera esposta ai raggi solari e quella immersa nelle tenebre. Cioè i punti giacenti su una medesima superficie sferica avranno pressione diversa secondo che appartengano alla ozonosfera esposta ai raggi solari oppure no; precisamente i punti appartenenti alla prima avranno pressione maggiore degli altri, perché la massa che sovrasta i primi è maggiore di quella che sovrasta i secondi. Di conseguenza, in alto si avrì un sistema di venti, che spirano dall'atmosfera esposta ai raggi solari verso quell'altra. Tali venti produrrammo un gradiente di pressione anche negli strati più bassi dell'atmosfera, ma diretto in verso opposto. Quindi in basso si avrà un sistena di venti spiranti dall'atmosfera immersa nelle tenebre verso l'altra.

Secondo i calcoli di Buttler e Small l'assorbimento delle radiazioni ultraviolette da parte dell'ozono fornisce circa il $60 \%$ della doppia oscillazione giornaliera misurata al suolo con i massimi coincidenti, con buona approssimazione, con quelli osservati. Il rimanente contributo potrebbe essere fornito dall'assorbimento del calore solare da parte del vapore acqueo e dell'anidride carbonica negli strati più bassi dell'atmosfera.

A prima vista il riscaldamento della ozonosfera da parte dei raggi solari ultravioletti dovrebbe produrre una oscillazione della pressione atmosferica terrestre con un periodo di 24 ore. Però ciò non è completamente vero, gracché, secondo i suddetti scienziati, ma analisi approfondita dell'effetto descritto sopra, fornisce - per il fenomeno di risonanza - una forte componente semidiurna. I'ampiezza della prima armonica di 12 ore dovrebbe essere almeno uguale alla oscillazione diurna di 24 ore. Mi pare che anche a questa ipotesi si possano muovere 
delle critiche, però ha il merito di fondarsi su dati sperimentali, che presentano un periodo di 12 ore. Tuttavia, anche secondo la conclusione, cui giungono Buttler e Small, l'ultima parola su tale argomento non è stata ancora detta, è quindi necessario eseguire ancora molte ricerche sperimentali e teoriche.

Questo articolo può essere considerato come la continuazione di quello pubblicato in "Geofisica e Meteorologia" (`). Già nel 1963, analizando i diagrammi del gradiente verticale del campo elettroatmosferico durante l'intervallo di tempo di sette amni, avevo ottenuto che il gradiente verticale del ampo elettro-atmosferico a Venezia e a Possagno (villaggio situato ai piedi del Monte Grappa a circa $300 \mathrm{~m}$ sul livello del mare), durante il tempo non perturbato dal punto di vista elettrico, presenta un andamento simile a quello della pressione atmosferica, cioè una doppia oscillazione diurna, con un periodo di circa 12 ore. Sono ritornato su tale argomento, studiandolo in maniera più approfondita, perché esso mi sembra di capitale importanza. Siccome la doppia oscillazione diurna della pressione atmosferica e del gradiente verticale del campo elettroatmosferico presenta un andamento molto simile a Venezia e a Possagno, sono giunto alla convinzione che tra $\mathrm{i}$ due fenomeni ci sia uno stretto legame. Tale legame potrebbe essere di doppio genere: o di causa ed effetto, cioè una delle due oscillazioni potrebbe essere prodotta dall'altra; oppure tutte e due potrebbero essere prodotte principalmente da una stessa causa diversa. Siccome lo studio separato della doppia oscillazione diurna della pressione atmosferica finora non ha trovato una spiegazione certa, mi pare che convenga studiare le due oscillazioni (della pressione e del gradiente del potenziale elettro-atmosferico) globalmente. Così le due oscillazioni divengono due aspetti del medesimo problema. Probabilmente una scoperta, anche di lieve entità, compiuta su una delle due oscillazioni, proietterà la sua luce anche sull'altra. Dopo oltre undici anni di misure del gradiente verticale del campo elettro-atmosferico a Venezia e per qualche mese a Possagno, e dopo aver osservato molti diagrammi delle medie orarie ottenuti in altre parti della superficie terrestre, sono griunto alla convinzione che se non ci fosse la componente locale, molto variabile da luogo a luogo, gli elettrogrammi ottenuti nelle più disparate parti della Terra, dovrebbero mostrare una doppia oscillazione diurna con il periodo di circa 12 ore simile a quella della pressione atmosferica. Effettivamente una doppia oscillazione diurna del gradiente verticale del campo elettroatmosferico è stata rilevata in diverse parti della Terra; però spesso i 
massimi ed i minimi non coincidono con quelli ottenuti da me. Tuttavia moltissime stazioni situate nelle più disparate parti della superficie terrestre, rivelano un minimo, molto accentuato, tra le ore 1 e le 6 ed un massimo tra le 18 e le 22 ; il massimo che dovrebhe aver luogo tra le 10 e le 12 ed il minimo tra le 16 e le 18 si manifestano in maniera più incerta.

In questi giorni ho visto con soddisfazione che già nel 1908 il prof. Inigi De Marchi riferisce che anche il gradiente verticale del campo elettro-atmosferico presenta ordinariamente ma doppia oscillazione diun'm (5).

Data l'importanza dell'argomento, adesso riferiro i risultati, a cui sono giunto dopo un lavoro molto accurato e, per quanto mi is stato possibile, esauriente. Tra gli elettrogrammi del quadriennio 19631966 ne ho scelto 122, che presentavano un andamento regolare; calcolando le medie orarie del gradiente verticale del campo elettroatmosferico di tali diagrammi ho traceiato i grafici riprodotti nel testo.

I diagrammi dei singoli mesi dimostrano in maniel'a certa che il minimo del mattino non può dipendere dal sorger del sole come del resto ia stato rilevato da altri $\left.{ }^{8}\right)$. Infatti se esso dipendesse dalla levata del sole, detto minimo dovrebbe spostarsi di circa due ore e mezzo dai giorni più brevi a quelli più lunghi, e quindi dovrebbe presentarsi più tardi nei mesi invernali rispetto a quelli estivi. Il che non avviene assolutamente. Per es. in Gennaio e Dicembre esso ha luogo alle $4^{\mathrm{h}}$, mentre a Luglio si presenta alle $5^{\mathrm{h}}$.

Particolare interesse presentano le due ultime Figure, che sintetizzano le caratteristiche fondamentali di tutti gli elettrogrammi presi in considerazione. La prima di esse ci rivela che in generale il gradiente verticale del campo elettro-atmosferico dal 1963 fino al 1967 ì andato continuamente aumentando, specialmente durante i due ultimi anni. La seconda rivela in modo evidente la doppia oscillazione diuma con il periodo di circa 12 ore. Inoltre tutti i diagrammi anmuali del quinquennio (1963-67) e il diagramma generale presentano un minimo principale alle $17^{\mathrm{h}}$ ed $\mathrm{mn}$ minimo secondario tra le $13^{\mathrm{n}}$ e le $12^{\mathrm{n}}$. Un confronto tra i diagrammi delle medie orarie del quinquemnio 1963-6i con gli analoghi del settennio $1956-1962$ ci mostra che tra i due gruppi di diagrammi esiste un buon accordo: solo c'è da osservare che i diagrammi del settennio 1956-62, presentano il massimo del mattino (tra le $10^{\mathrm{h}}$ e le $12^{\mathrm{n}}$ ) meno accentuato ed inoltre non contengono il minimo secondario tra le $13^{\prime \prime}$ e le $1.5^{\mathrm{h}}$. 

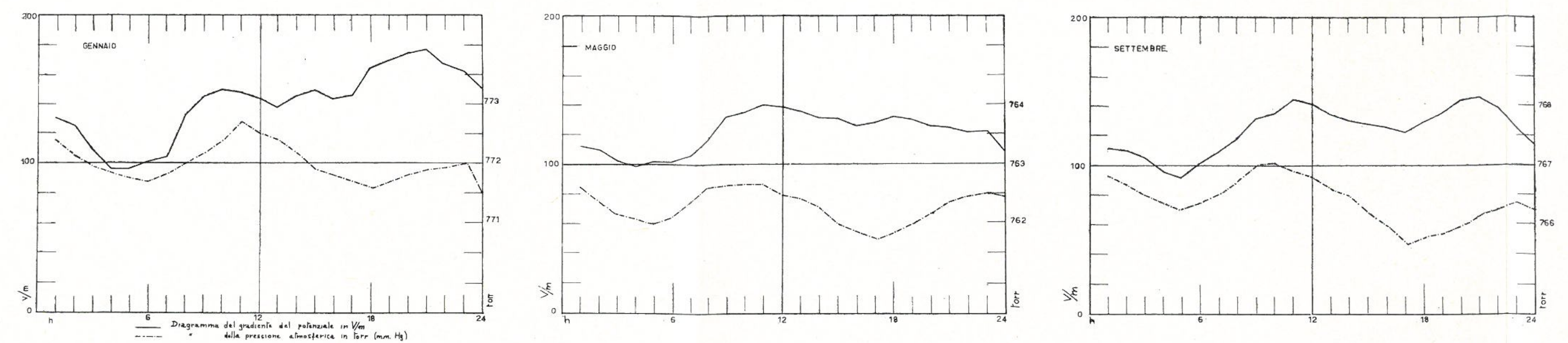

R. JANESELLI
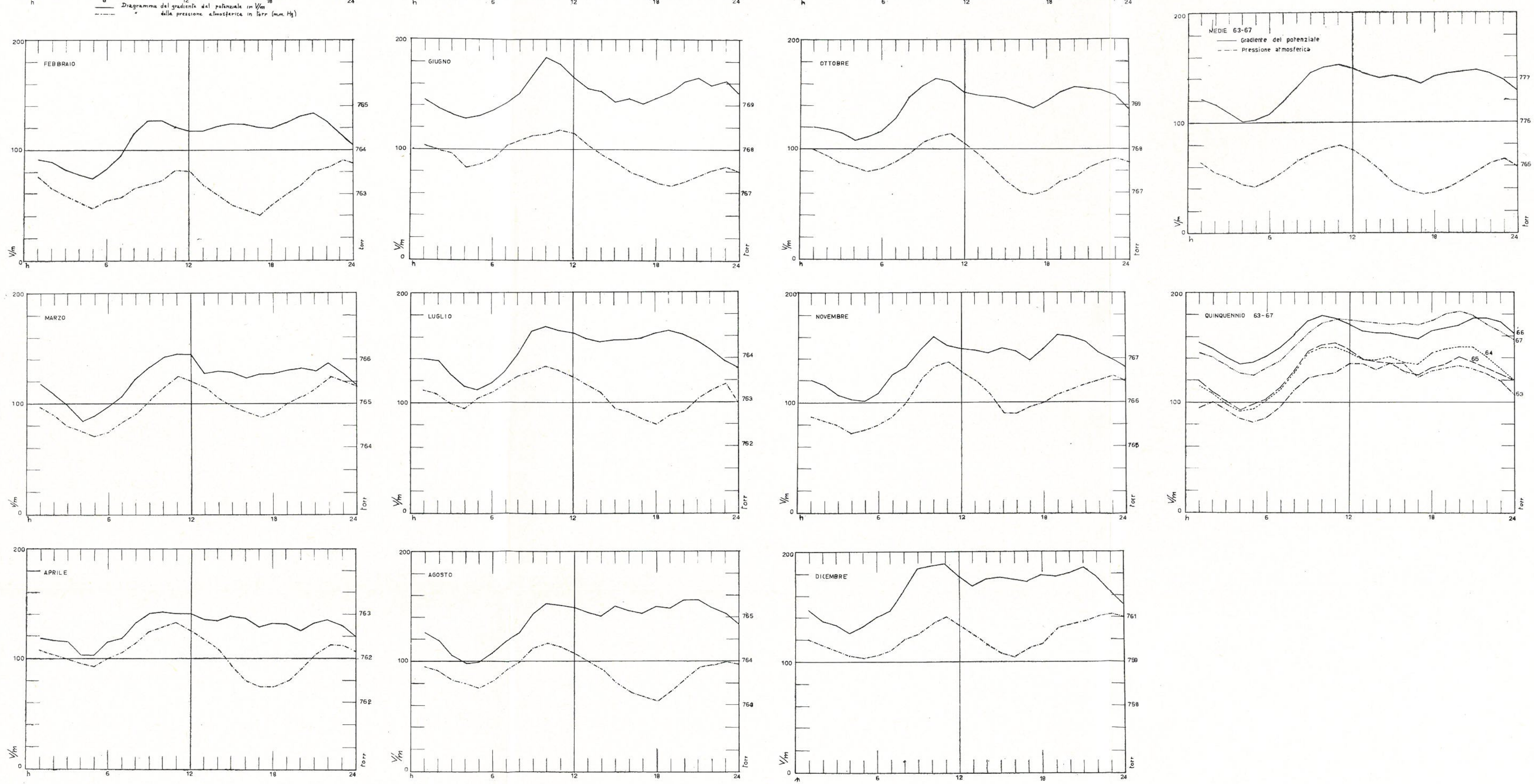
E interessante osservare che a Venezia la media generale del gradiente verticale del campo elettro-atmosferico per il quadriennio (1963-66) è $132 \mathrm{~V} / \mathrm{m}\left(^{*}\right)$.

Avendo a mia disposizione un grande numero di barogrammi tracciati dal barografo (S.I.A.P. - Modello grande) del mio Osservatorio, mi è sembrato opportuno compiere su di essi uno studio analogo a quello effettuato sopra gli elettrogrammi, di cui ho parlato prima.

I barogrammi presi in considerazione si estendono sopra un arco di tempo eguale a 9 anni dal 1959 al $196 \pi$. Ovviamente un intervallo di 9 anni non è uno spazio di tempo trascurabile; eppure, con mia grande meraviglia, ho potuto utilizzare solo 70 barogrammi (pochissimi in confronto del numero degli elettrogrammi). Infatti moltissimi di essi mostrano con evidenza la doppia oscillazione diurna; ma la maggior parte non potè essere da me utilizzata, perché la pressione da essi misurata era o in rapido ammento o in rapida diminuzione. Credo opportuno far osservare che sulla scelta dei barogrammi ho cercato di essere assolutamente obiettivo, non lasciandomi guidare da nessum preconcetto. Forse è la prima volta che si studia a Venezia la doppia oscillazione diurna della pressione atmosferica sperimentalmente; e quindi ovviamente ci tengo moltissimo che i diagrammi da me tracciati riproducano fedelmente la realtà obiettiva.

Effettuando sopra i barogrammi scelti le medesime operazioni eseguite sopra gli elettrogrammi, ho ottenuto le Figure del testo, nelle quali sono messi a confronto i diagrammi delle medie orarie del gradiente verticale del campo elettro-atmosferico e della pressione atmosferica. I diagrammi della pressione atmosferica mostrano con più regolarità degli altri la doppia oscillazione diurna con il periodo di circa 12 ore. Il che era da aspettarsi, giacché il campo elettro-atmosferico può essere perturbato da moltissime cause. Per es. a Venezia se l'aria contiene fumi provenienti dalle fabbriche di Marghera, anche in piccole quantità, il campo elettrico si presenta molto perturbato.

Anche qui dobbiamo escludere in maniera categorica che il minimo del mattino, il quale con più frequenza si presenta verso le $5^{\text {hn, }}$ dipenda dal sorgere del sole per le medesime ragioni addotte sopra riguardo al minimo giornaliero assoluto del gradiente del potenziale elettro-atmosferico. Qui credo necessaria una precisazione: il sorgere

(*) I calcoli relativi ai diagrammi mensili sono stati eseguiti verso la meta del 1967. Attualmente, a lavoro compiuto. si hanno anche i dati per lo stesso anno 1967 e pertanto se ne traccia, in firura, il diarramma relativo. 
ed il tramonto del sole agiscono sulla pressione atmosferica e sul gradiente verticale del potenziale elettro-atmosferico globalmente in quanto il sole agisce contemporaneamente sull'atmosfera situata nei due emisferi presa sul suo insieme. Ecco la ragione per cui il minimo del mattino ed il massimo della sera si presentano in tutte le stagioni, con buona approssimazione, alla stessa ora. Il parallelismo tra i due diagrammi mensili del gradiente del potenziale e la pressione è molto più accentuato durante la prima parte del giorno (dalle 0 alle 12 ore) che durante la seconda parte (dalle 12 alle 24 ore). Precisamente l'intervallo di tempo dove il parallelismo is meno perfetto va dalle $14^{\text {" }}$ alle 23 ".

Probabilmente cio si deve attribuire al fatto che durante il pomeriggio e le prime ore della sera il campo elettro-atmosferico frequentemente viene perturbato da moti convettivi e avvettivi di masse d'aria. Moltissime volte ho osservato che quando il vento cambia di direzione, il gradiente verticale del campo elettro-atmosferico subisce delle rapide variazioni, come ho grià scritto altre volte ("). Tale parallelismo è ancora molto più accentuato nei due diagrammi generali, che sintetizzano le caratteristiche fondamentali di tutti gli elettrogrammi e barogrammi presi in considerazione. L'unico disaccordo, degno di rilievo, è il massimo della sera, il quale per il gradiente del potenziale cade verso le $21^{11}$ e per la pressione atmosferica alle $23^{\text {h }}$ circa. Tale disaccordo si puo spiegare facilmente facendo qualche considerazione molto elementare sopra il clima di Venezia.

Dopo il tramonto del sole, con tempo bello, a Venezia cambia la direzione della brezza, la quale durante le ore di sole spira dal mare (da SE o da SSE) e durante le ore notturne da terra (da N). Tale cambiamento della direzione della brezza agisce sopra le due grandezze considerate in senso opposto: cioe fa diminuire il gradiente del potenziale (in quanto l'aria proveniente dal mare porta con sè una grande quantita di ioni positivi) e fa aumentare la pressione atmosferica per ovvie ragioni. Un'altra causa che impedisce alla pressione atmosferica di diminuire anche dopo le $22^{\text {n }}$ è il fatto che, logicamente, nelle prime ore della notte viene a cessare la colonna d'aria ascendente prodotta dal riscaldamento solare.

CENA SUL CAMPO ELETTRICO DELL'ATMOSFERA TERRESTRE.

Dopo le scoperte compinte in questi ultimi ammi intorno agli strati superiori dell'atmosfera terrestre, specialmente per mezzo di razzi e 
satelliti artificiali, pare giunto il momento di dover considerare il campo elettricó dell'atmosfera terrestre sot to un punto di vista globale. Infatti i diversi strati dell'atmosfera terrestre, incominciando dalla troposfera per terminare con la ionosfera e la esosfera non sono separati tra di loro da diaframmi impermeabili; ma masse imponenti di gas passano continuamente da mo strato ad un altro. Orviamente tali trasporti sono prodotti specialmente dalle maree atmosferiche. Anzi secondo qualche geofisico ${ }^{\left({ }^{10}\right)}$ la struttura generale del campo eletirico dell'atmosfera terrestre avrebbe la sua origine basilare nella separazione di carica elettrica nella mesosfera e nella ionosfera più bassa. Tale separazione di carica sarebbe poi prodotta da venti verticali eausati dalle maree atmosferiche. Questi venti verticali darebbero origine ad una separazione di carica elettrica, perché i portatori di carica da essi trasportati hammo uma diversa mobilita a causa della loro massa molto differente. Pare accertato rhe movimenti verticali provocati da circolazioni di marea nell'emisfero esposto ai raggi solari durante il mattino e le prime ore del pomeriggio, diano origine ad una separazione di carica elettrica in direzione verticale con cariche positive spinte a muoversi verso il basso. Infatti gli ioni positivi sono trascinati dal vento verticale verso il basso per effetto degli urti con le molecole del gas: mentre gli elettroni-che hammo massa molto minore-non sono influenzati da tale vento verticale ma sentono, naturalmente, oltre il campo geomagnetico, il campo elettrico risultante da tale separazione di cariche. Questo processo di separazione di curica elettrica produrra un campo elettrico, che manterad, in maniema rigida, una carica spaziale positiva nella stratopausa ed una negativa nella ionosfera più bassa durante il mattino e le prime ore del pomeriggio. Tale separazione di carica elettrica avrà come risultato la produzione di campi elettrici e di correnti elettriche, che modificano la ionosfera " normale" in accordo con la struttura elettromagnetica osservata. Saturalmente tale struttura elettrica globale diuma si trovera sempre sotto il controllo della circolazione prodotta dalle maree atmosferiche.

Come i noto, sopra i $60 \mathrm{~km}$ d'altezza, l'atmosfera terrestre è formata in gran parte da ioni, specialmente da elettroni. Ovviamente questo gas, in conseguenza della sua grande ionizzazione, si comporterà come un buon conduttore di corrente elettrica, e quindi quando si muove nel campo magnetico terrestre, verramno generate nel suo interno delle correnti indotte, chimate commemente correnti dinamo. Inoltre è noto che la corrente elettrica generata internamente ad $\mathrm{m}$ gas da un campo elettrico ortogonale ad un campo magnetico, non si 
muove nella medesima direzione del campo elettrico; compare cioè ma componente di corrente, che ì perpendicolare sia al campo elettrico sia al campo magnetico. Tale componente di corrente comunemente viene denominata "corrente di Hall" ("1).

Verso gli $80 \mathrm{~km}$ d'altezza, le correnti dinamo in prevalenza saramno formate da elettroni, i quali, secondo i dati di osservazione, dalle $4^{\mathrm{h}}$ del mattino fino alle prime ore del pomeriggio (alle $14^{\mathrm{h}}$ (irca) si muovono verso Est. Quindi, alle basse latitudini, l'emisfero esposto ai raggi solari della regione Est si trova costantemente ad un potenziale più elevato rispetto alla regione nottuma, con il massimo verso le

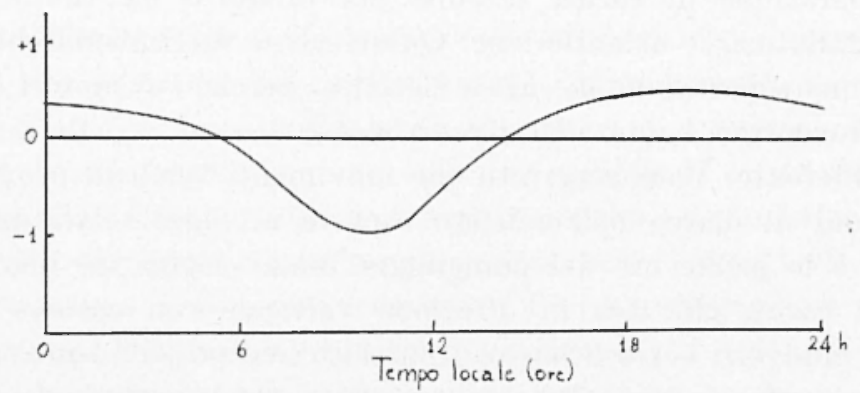

Fig. 2 - Caratteristica diurna dei moti verticali attraverso la mesopansa a $15^{\circ}$ di latitudine (da "The source of atmospheric electrification" by Willis L. Welub.).

X. B. - Quale unità di misura sull'ordinata, si è preso il massimo negativo della curva.

ore 14 ed il minimo verso le ore 4 . A partire dalle prime ore del pomeriggio (1.th circa) lino alle $t^{\text {th }}$ del giorno seguente, nelle regioni di bassa latitudine i moti verticali del gas ionizzato prodotti dalle maree atmosferiche cambiano di verso e quindi muteranno di verso anche le correnti dinamo. Di conseguenza le correnti elettroniche di Hall (*) si muoveranno verso Ovest. Però come si vede dalla Fig. 2, la quale rappresenta l'andamento dinamo dei moti verticali attraverso la mesopausa a $15^{\circ}$ di latitudine, i venti verticali verso l'alto sono molto meno accentuati di quelli verso il basso e cosi le variazioni di velocita sono piuttosto piccole, specialmente durante le ultime ore della sera e le prime ore della notte (dalle $19^{\mathrm{h}}$ alle $4^{\mathrm{h}}$ ). Di conseguenza la densità degli elettroni nella regione Est diminuira lentamente durante tale pe-

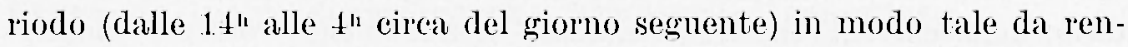

(*) Cfr. Writis I. Weвв., The Source of Atmospleric Blectrification. 
dere inefficiente il mecomismo che genera la corrente di Itall, durante le nltime ore della notle.

E poi evidente che ma separazione di carica elettrica può essere generata anche da circolazioni atmosferiche orizzontali. In questo caso, se la circolazione generale avviene verso Est, la corrente dinamo formata da elettroni) si moverà lungo piani verticali e precisamente sarà diretta verso lalto. Invece, se la circolazione generale arviene verso Ovest, la corrente dinamo sarà diretta verso il basso. Tale processo di separazione di carica sana poi globalmente asimmetrico, in quanto sarà molto più efficiente nell'emisfero esposto ai raggi solari che nell'altro emisfero, per la maggior concentrazione di elettroni e ioni positivi. Quando il vento proviene da Ovest, il che ordinariamente, avviene durante i mesi invernali, la corrente prodotta dalla circolazione dei venti avà la medesima direzione della corrente generata dalle maree atmosferiche: cosicche la corrente prodotta dalla circolazione generale invernale fara ammentare la corrente dinamo nel circuito del mattino, mentre farà diminuire l'intensita di tale corrente nel circuito della sera.

Al contrario quando il vento proviene da Est, il che generalmente ha luogo durante i mesi estivi, la circolazione prodotta dai venti e quella generata dalle mare atmosferiche avramo direzione opposta durante il mattino e la medesima direzione durante la sera. Di conseguenza si avrà una diminuzione nell’intensità della corrente dinamo durante il mattino e un ammento durante la sera.

Qui credo necessario precisare meglio quale è la circolazione generale dei venti dell'atmosfera terrestre. Nella parte più bassa dellatmosfera, alle medie latitudini, esiste un sistema di renti, che provengono sempre da Ovest sia durante i mesi invernali che quelli estivi. Tale sistema di venti, alle volte chiamato "jet stream ", serpeggia attorno alla terra per tutto l'anno, però con più forza durante linverno, e si estende fino a circa $2.2 \mathrm{~km}$ d'altezza.

Sopra tale altezza, sempre alle medie latitudini, durante i mesi invernali si presenta un altro sistema di venti pure provenienti da Ovest, il cui nucleo principale oscilla tra i jo e i $60 \mathrm{~km}$ d'altezza. La velocita di tali venti a grande altezza d̀ di circa $250 \mathrm{lkm} / \mathrm{h}$, cioc superiore al doppio della velocita media del sistema inferiore. Peró questo secondo sistema di venti dumante $i$ mesi estivi cambia di direzione e precisamente soffia da Est. Quindi i due sistemi di venti durante i mesi invernali provengono dalla stessa direzione, cioè da Ovest, mentre durante i mesi estivi provengono da direzioni opposte e precisa- 
mente quello inferiore proviene da Ovest e quello superiore proviene da Esit $\left({ }^{12}\right)$.

Ovriamente tale diverso (omportamento dei suddetti sistemi di renti dovebbe far sentire la sua influenza anche sul gradiente verticale del campo elettro-atmosferico al suolo, specialmente in montagna.

Questa potrebbe essere la ragione, per cui a Sauze d'Oulx (Alpi Occidentali Italiane a m 1865) l'aumento del gradiente del potenziale elettro-atmosferico durante il mattino e le prime ore del pomeriggio (15 h circa) dei mesi invernali is molto più rapido e considerevole che durante il medesimo intervallo di tempo dei mesi estivi $\left({ }^{13}\right)$.

\section{CONCLUSIONE (*).}

Hi sembra poco logico afiemane che sia casuale il fatto che in molti luoghi della Terra la ionosfera più bassa, la pressione atmosferica

(*) Giunto verso la fine del presente articolo. ho letto con molto interesse ma Memoria dal titolo: "Risultati delle Osservazioni sull'blettricismo Atmosferico istituite nel R. Osservatorio di Modena nseritta dal Prof. Domenico Ragona e pubblicata nelle "Memorie della R. Aec. di Scienze di Iodena", Tomo XI, 1870. In tale memoria il Ragona riferisce di avere esegruite a Modena nel 1868 e 1869 molte misure sia del gradiente del potenziale elettro-atmosferieo sia delle conrenti telluriche. Per misurare il erradiente del potenziale elettro-atmosferieo il fisieo di IIodena adoperava un eleftrometro hifilare e, non avendo a sua disposizione un registratore, exegruiva sette misure al giorno (anche di notte). Costruenclo il diagramma delle medie orarie (pubblicato nella suldetta Memoria) egli otteme un grafico, che presenta una doppia oscillazione giomaliera, identico al corrispondente della pressione atmosferica. Secondo il Ratrona la doppia oscillazione giornaliera della pressione atmosferica dipenderebbe da due canse: $1^{\circ}$ ) - dal calore solare che darebbe origine ad ma oscillazione regolare diuma com un massimo ed un minimo; $2^{\circ}$ ) - lalla elettricita atmosferica (gradicnte del potenziale) la quale darebbe origine ad una doppia oscillazione giomaliera con due massimi e due minimi.

Juoltre il Ragona osserva che il barometro non indiea solo le variazioni di peso dellatmosfera, ma anche quelle dell elasticità dell'aria (cioc dellelettricita di questa). Riporta anche aleune parole seritte dal sig. Rocaut nel 1770 nel Joumal Encyclopedique: "Le barometre annonce plutôt ou le moins de fluide electrique libre dans lair, que le plus ou le moins de poids de la colonne d'air qui y correspond". Inoltre riferisce che nelle antiche memorie dell'Accatemia Imperiale di Bruxelles (Tom. Io) si trova uno seritto molto pregevole dell'Abate Ianu, in cui si dimostra che 
ed il gradiente verticale del campo elettro-atmosferico presentino il minimo valore diurno alle $4^{\mathrm{h}}$ circa, naturalmente con tempo bello e non perturbato sia dal punto di vista barico che da quello elettrico; cosi pure il fatto che il vento verticale diretto dalla ionosfera alla stratopausa, la pressione atmosferica ed il gradiente verticale del campo elettro-atmosferico raggrimgano il loro valore massimo diurno assoluto o relativo alle $10^{\mathrm{h}}$ circa. Inoltre è da osservare che a Venezia il parallelismo tra tutti i diagrammi of tenuti dalle medie della pressione atmosferica e del gradiente verticale del campo elettro-atmosferico is quasi sempre perfetto dalle $23^{\text {h }}$ lino alle $14^{\text {h }}$ circa (cioe per buona parte della notte e del giorno, per circa 10 ore). T'altra parte ì da ricordare che il potenziale della ionosfera più bassa raggiunge il suo massimo valore alle $14^{\mathrm{h}}$ circa, dopo di che diminuisce lino alle $4^{\mathrm{th}}$ circa del griorno seguente.

I sistemi di venti verticali e orizzontali, di cui si è parlato sopra, soffiano in $u n$ verso dalle $4^{\text {h }}$ fino alle $1^{\text {th }}$ circa e quindi nel verso opposto dalle $14^{\text {h }}$ fino alle $t^{\text {h }}$ del giorno seguente, cioè spirano in un verso per 10 ore circa e nel verso opposto per le altre 14 ore del giolno. Ni pare che il sistema di venti preso in considerazione dai due scienziati anstraliani si possa considerare come un caso particolare di nostro sistema più generale. Siccome latmosfera della Terra possiede un periodo di risonanza, con approssimazione uguale a 1.2 ore, è logico pensare che tali sistemi di venti facciano sentire la loro influenza anche negli strati più bassi della troposfera, e quindi generino nella pressione atmosferica e nel gradiente del potenziale elettro-atmosferico delle oscillazioni aventi un periodo di circa 12 ore. Per spiegare la coincidenza riferita sopra, mi pare ovvio ammettere che il sistema dei venti prodotti dalle maree atmosferiche facciano sentire la loro intluenza sopra la pressione atmosferica ed il gradiente del potenziale elettroatmosferico, anche negli strati più bassi della troposfera, pure per mezzo dei campi elettrici da essi generati (*). Verso le $4^{\text {th }}$ la pressione atmosferica ed il gradiente del potenziale elettro-atmosferico assumono il minimo valore, perché in tale ora la stratopausa presenta il minimo

"les variations du barometre dépendent bien plus dell'elasticite de laar. que des variations de pesanteur dans l'atmosphere entieren. Evidentemente i due passi riportati in francese contengono delle esagerazoni. però mi pare che esprimano un fondo di verità.

(*) Ovviamente tali venti possono agire sopra le due grandezze considerate anche in altri modi. 
valore del potenziale. Esse vanno continuamente aumentando, finché cresce la velocità del vento verticale diretto verso il basso, cioc fin verso le $10^{\mathrm{h}}$. Queste due grandezze non aumentano fino alle $14^{\text {he }}$, perché il calore solare (con tempo bello) riscalda grli strati più bassi della troposfera e quindi produce una colomma d'aria ascendente, che traseina con se una grande quantita di ioni positivi situati per l'effetto elettrodo in vicinanza del suolo: la qual cosa fa dimimuire sia la pressione atmosferica come il gradiente del potenziale elettro-atmosferico. Il parallelismo tra la variazione diuma della pressione atmosferica e il gradiente del potenziale elettro-atmosferico pare confermato dai diagrammi ottenuti in montagna. Per es. il massimo diurno della pressione atmosferica, che a Venezia ed in molti altri luoghi si presenta tra le $10^{\mathrm{h}}$ e le $11^{\mathrm{h}}$, sul Monte Rosa si sposta verso le $15^{\mathrm{h}}-16^{\mathrm{h}}$ (Colle d'Olen, m 290-1: Capanna Margherita, $n$ 4560). Tale massimo del gradiente del potenziale elettro-atmosferico si presenta verso le $15^{\mathrm{h}}$ sia sulla sommitì del Monte Fuje (m 3756) in Giappone sia a Sauze d'Aulx (m 1865), situato sulle Alpi Occidentali Italiane. Da ciò sembra di poter concludere che la carica elettrica positiva accumulata nella stratopausa dai venti verticali sopra descritti, faccia sentire meno la sua influenza sopra gli strati più bassi della troposfera rispetto a quelli alquanto più alti, come del resto era da aspettarsi.

Inoltre ì da sottolineare che le registrazioni effettuate molti anni fa, specialmente se compiute in città industriali, rivelano con più evidenza la doppia oscillazione giomaliera del gradiente del potenziale elettro-atmosferico di quelle eseguite in questi ultimi decenni. Per es. a Tokyo il diagramma delle medie orarie di tale parametro nell'intervallo di tempo 1897-1903, mostra con la più grande evidenza tale doppia oscillazione giornaliera; mentre nell'intervallo di tempo GennaioAgosto 1962 essa è appena accennata. Il massimo della sera, anticipato di qualche ora, è quasi completamente scomparso. Evidentemente ciò si deve attribuire all'inquinamento atmosferico prodotto specialmente dalle fabbriche e dalle automobili. Sotto questo aspetto Venezia si trova in una situazione del tutto privilegiata.

Forse il tallone di Achille della nostra ipotesi è il massimo della sera, che si presenta in moltissimi barogrammi ed elettrogrammi in ogni luogo della Terra. Mi pare di poter spiegare il massimo della sera nel seguente modo: quando la ozonosfera non è più colpita dai ragroi solari, essa incomincia a contrarsi; inoltre verso i $75 \mathrm{~km}$ d'altezza esiste un sistema di venti, che al tramonto del sole, cambia di direzione. Come si è visto sopra, i venti spirano dalla ozonosfera esposta 
ai raggi solari verso laltra parte immersa nelle tenebre. Per di più lo strato $\mathrm{D}$, al sopraggiungere delle tenebre sparisce improvvisamente. Tutto ciò ci porta a concludere che, al tramonto del sole, nello strato d'atmosfera compreso tra i $40 \mathrm{~km}$ e i $75 \mathrm{~km}$ daaltezza debbano avvenire dei fenomeni veramente grandiosi, specialmente nelle zone aurorali, come del resto ci viene dimostrato dai dati d'osservazione. is logico che tutti questi fenomeni, del tutto eccezionali, facciano

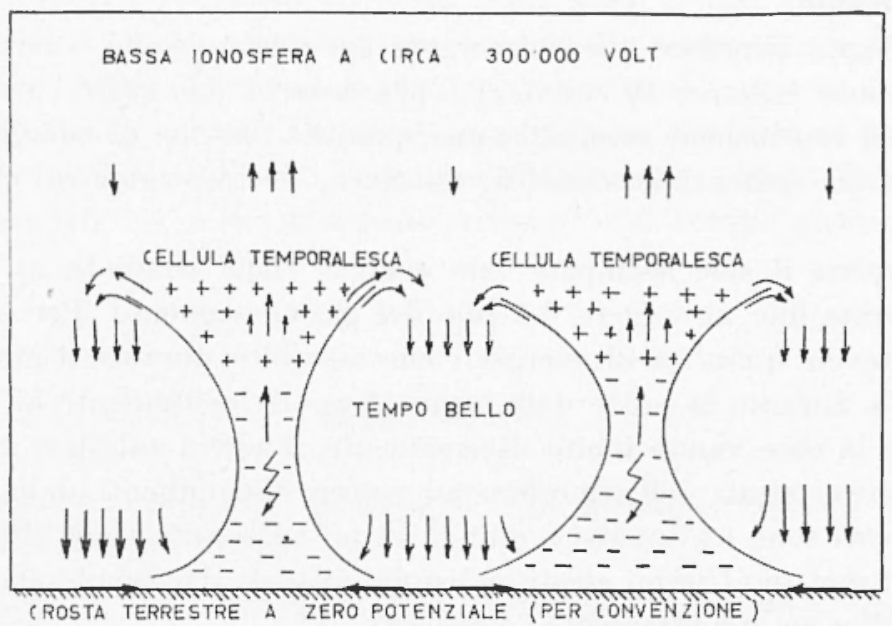

Fig. 3 - Schema che mostra la relazione che lega l'attività temporalesca con il campo elettrico di bel tempo. La frece indicano il senso della corrente elettrica.

sentire la loro influenza sugli strati immediatamente sottostanti, specialmente sulla stratopausa. Nella zona di separazione tra la regione esposta ai raggi solari e quelia immersa nelle tenebre dovrebbe formarsi una specie di fronte, che si propaga attraverso l'atmosfera con il moto apparente del sole. Questa potrebbe essere la ragione, per cui la massima frequenza dei temporali, che si abbattono sulla superficie terrestre presa globalmente, si presenta verso le $19^{n}$. Essendoci poi un intimo legame tra l'attivita temporalesca ed il gradiente del potenziale elettro-atmosferico di bel tempo, ovviamente quest'ultimo dovrebbe presentare il suo massimo valore quando l'attivita temporalesca è massima (Fig. 3). Inoltre, al tramonto del sole, nello strato di atmosfera compreso tra la stratopausa e i $60 \mathrm{~km}$ d'alteza circa, per la contrazione della ozonosfera si produrranno dei venti verticali diretti verso il basso, la quale cosa darà origine ad una separazione 
di carica elettrica e precisamente ad un trasporto di carica, prevalentemente positiva nella stratopansa. Il che farà ammentare l'intensità della corrente di bel tempo diretta verso il basso, il gradiente del potemziale eletto-atmosferico e la pressione atmosferica al suolo.

A prima vista potrebbe forse sembrare che un fenomeno simile dovesse presentarsi al sorgere del sole. Ma un esame nu po' approfondito ci mostra che le cose al sorgere del sole devono andare molto diversamente.

Bisogna ricoudare che dal sorgere del sole (alle ore 5 circal fino al tramonto (alle ore 19 circa), cioè per circa 14 ore, tutta l'atmosfera presa nel suo insieme accumula una quantita enome di energia sotto varie forme (potenziale, cinetica, calorifica, elettromagnetica, chimica, ece.).

Appena il sole scompare tale energia viene irradiata in moltissime forme fino al sorgere del sole del giorno seguente. Per esempio una notevole quantita di energia viene assorbita durante il giorno ed irradiata durante la notte dalla ozonosfera. Evidentemente al sorgere del sole le cose vanno molto diversamente. Basterì riflettere che alla sera i primi strati dell'atmosfera ad essere abbandonati dalle radiazioni solari sono i piu bassi e quindi un po' alla volta i piu alti. Viceversa al mattino i primi strati ad essere investiti dai raggi solari sono i più alti e poi gradatamente i più bassi.

Concludendo possiamo aftermare che la doppia oscillazione griornaliera della pressione atmosferica e del gradiente verticale del campo elettro-atmosferico ì prodotta dalle maree atmosferiche e da altre cirrolazioni secondarie di venti, le quali, alla loro volta, sono generate in prevalenza dal calore solare. Inoltre sembra rerto che le suddette grandezze siano influenzate contemporaneamente da rampi eletrici esistenti nell'atmosfera terrestre; il gradiente del potenziale elettroatmosferico per ovvie ragioni: la pressione atmosferica per il fatio che anche gli strati piu bassi della troposfera contengono sempre una grande quantita di ioni, i quali evidentemente possono essere attatti o respinti dai campi elettrici dell'atmosfera terrestre. Queste attrazioni o repulsioni, in certi casi, possono assumere dei valori veramente notevoli specialmente durante i temporali. Probabilmente le rilevanti e rapide variazioni segnalate dai barogrammi durante i temporali sono dovute, oltre che alle imponenti colonne d'aria ascendenti e discendenti, alle eccezionali attrazioni e repulsioni esercitate sugli ioni da parte dei campi elettrici generati dalle nubi temporalesche. Inoltre i logico pensare che notevoli variazioni della pressione atmosferica, special- 
mente se rapide possano produrre delle oscillazioni del gradiente verticale del campo elettro-atmosferico. Inunque dalle relazioni, che legano la pressione atmosferica ed il gradiente verticale del campo elettroatmosferico con le maree atmosferiche ed i campi elettrici esistenti nell'atmosfera terrestre e dai mutui legami, che intercorrono tra le due grandezze, sembra di poter concludere che esse debbano presentare, con tempo bello e non perturbato, un andamento giornaliero simile.

Il quadro elettrodinamico degli strati bassi e medi dellatmosfera terrestre, qui appena abbozzato in forma molto schematica e sutetica, non ha la pretesa di essere completo, però ho fiducia che esso ci indichi la strada, lungo la quale bisognerà incamminarsi per trovare una spiegazione soddisfacente della doppia oscillazione giornaliera della pressione atmosferica e del gradiente verticale del campo elettro-atmosferico.

\section{BIBIJOGRAFIA}

(1) Enenta F., Lezioni di Meteorologia e di Aerologia. Citti di Castello, Casa Ed. Lapi, (19+1).

(-) Butres S. T', Atmospheric Tides. Scientific "American ", December, 1962.

(3) Panumo A., Sulle Maree atmosjeriche. "Atti XV Convegno Annuale. Ass. Geof. It. Roma, 16-17 Giugno $1966 \%$.

(') Lonv Kervin ('Thomson W.), On the Acceleration of the Earth's Rotation. "Proc. Roy. Soc. Edinburgh ", 11, (1882).

(5) De Mascui L., Teoria della doppia oscillazione diuma del barometro. "Atti R. lstituto Veneto di Scienze, Isettere ed Arti". (1908).

(i) SMan, K. A., BuTruen S. T., The semidiurnal Atmospherie Oseillation. "Journal of Geophysical Research", 66, 11, (Nov. 1961).

(i) Janesend R., Risultati di sette anni di osservazioni sul gradiente rerticale del campo elellro-atmosferico a Venezia. "Geofisica c Meteorologia ", XIII, 1-2, (I964).

${ }_{(8)}^{8}$ Gnenzr F. K., Amospheric Electricity at Brébeuf College Gieophysical Observatory in Montreal, Canada. "Cure and applied Geophysics ", 67, (1967).

(') Javeseiti R., Ricerche sul gradiente verticale del campo eleltro-atmos/erico a Venezia. "Atti Ist. Veneto Scienze, Isettere ed Arti ",'Tomo CXXII. $(1963-(34)$. 
(10) Iитакшиман H., Atmospheric Electricily Research in the Far East. Coronili s. C. Problems of Almospheric and Space Electricily. Elsevier Publishing Company, New York, 1965.

(11) Katokmax I. H., Elements of Magnegasdynamics. WV. B. Saunders Company, Philadelphia, 1967.

(12) R. E. Nowsil, The Circulation of the Epper Atmosphere. Sc. Am. March $196 \mathrm{jt}$.

$\left({ }^{13}\right)$ Bossolasco M. e Coll. The electro-atmospheric potential gradient at Sauz, dOulx: (Italian Western Alpes). "Geofisica e Meteorologia". XVI 111. $5-6(1967)$. 